\title{
Capacity of MIMO Channels: Asymptotic Evaluation Under Correlated Fading
}

\author{
Xavier Mestre, Student Member, IEEE, Javier R. Fonollosa, Senior Member, IEEE, and Alba Pagès-Zamora
}

\begin{abstract}
This paper investigates the asymptotic uniform power allocation capacity of frequency nonselective multiple-input multiple-output channels with fading correlation at either the transmitter or the receiver. We consider the asymptotic situation, where the number of inputs and outputs increase without bound at the same rate. A simple uniparametric model for the fading correlation function is proposed and the asymptotic capacity per antenna is derived in closed form. Although the proposed correlation model is introduced only for mathematical convenience, it is shown that its shape is very close to an exponentially decaying correlation function. The asymptotic expression obtained provides a simple and yet useful way of relating the actual fading correlation to the asymptotic capacity per antenna from a purely analytical point of view. For example, the asymptotic expressions indicate that fading correlation is more harmful when arising at the side with less antennas. Moreover, fading correlation does not influence the rate of growth of the asymptotic capacity per receive antenna with high $E_{b} / N_{0}$.
\end{abstract}

Index Terms-Correlated fading, free probability, multipleinput multiple-output (MIMO) capacity, random matrix theory.

\section{INTRODUCTION}

$\mathbf{I}$ T HAS traditionally been accepted that the use of multiple antennas at basestations in mobile communications scenarios increases their coverage and spectral efficiency, allowing for ever higher data rates and a lower power consumption. Recently, the advent of third-generation (3G) mobile communications standards has brought to consideration the use of multiple antennas at both mobile terminals and basestations. This option had been disregarded in previous mobile communications standards, due basically to the limited size of the handsets and the low frequency band of operation. Nowadays, the proliferation of wireless local area networks and the need for sophisticated and bandwidth-consuming services such as video over the radio interface has motivated the consideration of multiple antennas at both sides of the communications link.

The consequence of all this has been an increasing interest in the characterization of multiple-input multiple-output (MIMO)

Manuscript received May 1, 2002; revised November 1, 2002. This work was supported in part by the European Union through its program IST, the Spanish Government TIC2002-04594-, TIC2001-2356-, TIC2000-1025-C02-01, and in part by the Catalan Government (CIRIT) 2001SGR-00268. This paper was presented in part at the European Signal Processing Conference, Tolouse, France, 2002.

$\mathrm{X}$. Mestre is with the Centre Tecnològic de Telecomunicacions de Catalunya, Ed. Nexus, Campus Nord UPC 08034 Barcelona, Spain (e-mail: xavier.mestre@cttc.es).

J. R. Fonollosa and A. Pagès-Zamora are with the Department of Signal Theory and Communications, Universitat Politècnica de Catalunya, Campus Nord UPC 08034 Barcelona, Spain (e-mail: javier.fonollosa@upc.es; alba@gps.tsc.upc.es).

Digital Object Identifier 10.1109/JSAC.2003.810352 channels and in particular the actual data rates that they can support (at least, theoretically). Several papers (e.g., [1] and [2]) have shown that the channel capacity of a MIMO channel can increase as much as linearly with the minimum number of antennas used at either the transmit or receive side. The usual approach to reach this conclusion is to consider a random model for the channel (usually a frequency nonselective Rayleigh-fading distribution) and evaluate the mean value of its capacity averaging with respect to the channel statistics. Unfortunately, one does not generally obtain a closed form analytical solution using this procedure and asymptotic approximations have to be considered.

Here, we analyze the asymptotic capacity when the number of transmit and receive antennas are driven to infinity at the same rate. This might seem an oversimplification of the model, but in practice it is experimentally observed that these type of limits are more representative of the nonasymptotic reality than traditional asymptotic approaches, where only the number of antennas at one side of the communications link is assumed to grow. In our case, the ratio between transmit and receive antennas is held constant and consequently the asymptotic results turn out to be "less biased." The appropriate tool for dealing with these bidimensional limits is Free Probability Theory, a probability theory for noncommutative random variables introduced by Voiculescu in, e.g., [3], and [4]. This theory turns out to be very useful to describe the distribution of the eigenvalues of random matrices when their dimensions increase without bound.

In this paper, we will use some of the results of Free Probability Theory to investigate the influence of fading correlation at one side of the communications link on the actual MIMO asymptotic capacity. We propose a fading correlation function depending on a single parameter that measures the degree of fading correlation between antennas. In principle, this fading correlation function does not arise from a physical model and is introduced merely for mathematical convenience. In practice, though, it is observed that the correlation shape is very close to an exponentially decaying model. Based on this model, we give a closed form expression for the asymptotic capacity of the MIMO channel that depends explicitly on the aforementioned fading parameter. From that expression we try to shed some light on the influence of fading correlation on the asymptotic capacity per antenna from a purely analytical point of view. Of course, since we are restricting ourselves to a particular shape of the fading correlation function, we are losing some generality in the final expression. This seems to be the tradeoff for obtaining a closed form solution for the asymptotic capacity. 
The rest of the paper is organized as follows. Section II presents the signal model and the basic assumptions made in the paper. Section III derives the asymptotic capacity per antenna in the correlated fading model and Section IV analyzes the effect of fading correlation in different asymptotic situations. Finally, Section $\mathrm{V}$ presents a numerical validation of the theoretical results derived and Section VI concludes the paper.

\section{Signal Model And Channel CAPACiTy}

We focus on a general MIMO channel model with $M$ inputs and $N$ outputs, where the output signal can be modeled as a column vector

$$
\mathbf{y}=\sqrt{\varphi \mathbf{H s}}+\mathbf{n}
$$

with $\varphi \in \mathbb{R}$ a power gain factor, $\mathbf{H}$ an $N \times M$ channel matrix with complex entries, $\mathbf{s}$ a column vector containing the signals transmitted by the $M$ inputs, and $\mathbf{n}$ the noise component at the input of the receiver, which is modeled as a circularly-symmetric complex Gaussian distributed random vector with zero mean and covariance $E\left[\mathbf{n n}^{H}\right]=\sigma^{2} \mathbf{I}_{N}$ (here, $(\cdot)^{H}$ denotes transpose conjugate and $\mathbf{I}_{N}$ the $N \times N$ identity matrix). We will assume that perfect channel state information is only available at the receiver, i.e., the channel realization is perfectly known only at the receiving stage and is unknown to the transmitter. For a similar analysis but in terms of the "closed-loop" capacity (i.e., the capacity when the channel state information is available at both the transmitter and the receiver), the reader is referred to [5].

As explained above, it is interesting to evaluate the actual capacity in terms of bps/Hz that a MIMO system can support. Several authors have considered this problem: for instance, in [1] and [2] the channel matrix entries are modeled as independent circularly symmetric random variables with zero mean and unit variance and the mean capacity under an average global transmitter power constraint and uniform power allocation is shown to be the expected value (in terms of the channel statistics) of

$$
C(\beta, M, N)=\log _{2} \operatorname{det}\left[\mathbf{I}_{N}+\frac{\beta}{M} \mathbf{H H}^{H}\right]
$$

where $\beta$ is the quotient between the average received signal power and the noise power at each of the receivers. ${ }^{1}$ If we denote by $\left\{\gamma_{i}\right\}_{i=1 \ldots N}$ the eigenvalues of $N^{-1} \mathbf{H H}^{H}$ (with repetitions, if there are multiplicities higher than one), we can express

$$
C(\beta, M, N)=\sum_{i=1}^{N} \log _{2}\left[1+\frac{N}{M} \beta \gamma_{i}\right]
$$

and the capacity would be obtained after averaging with respect to the eigenvalues statistics. Unfortunately, as shown in [2, Th. 2] the general expression for this capacity does not seem to have a closed analytical form and the actual formula should be evaluated using numerical integration. For this reason, several authors have considered the asymptotic expression of such capacity, i.e., the capacity when the two dimensions of the MIMO system increase without bound at the same rate $(M, N \rightarrow \infty$,

${ }^{1}$ If $P$ denotes the total transmitted power, then $\beta=\varphi P / \sigma^{2}$.
$M / N=c$ with $0<c<\infty)$. For example, in [6] and [7] a closed form expression for the asymptotic capacity per antenna in the uncorrelated model was derived. Later, this was generalized to include correlations at the transmit and receive sides in [8], but the considered scenario was so general that no closed form expression for the capacity was obtained. The asymptotic MIMO capacity in the presence of random Gaussian interference has recently been analyzed in [9]. In all these contributions, the asymptotic evaluation is based on random matrix theory and the study of the eigenvalue empirical distribution function (i.e., the eigenvalue counting function $F^{N}(x)=\#\left\{\gamma_{i}^{N} \leq x\right\} / N$, with $\gamma_{i}^{N}$ the eigenvalues of the $N \times N$ matrix and $\#\{\cdot\}$ denoting the cardinality of a set) when the matrix dimensions increase without bound. The basic concept behind this approach is that, as the dimensions of the random matrix are driven to infinity, the empirical distribution function of the eigenvectors of some random matrix models tends to a nonrandom quantity. For the case considered above, where the entries of $\mathbf{H}$ are independent and identically distributed (i.i.d.) circularly symmetric Gaussian-distributed with zero mean and unit variance, it has been shown (cf. [10]) that the empirical distribution function of the eigenvalues of $N^{-1} \mathbf{H H}^{H}$ tends almost surely to the so-called Marčenko-Pastur distribution $F(x)$, corresponding to a density ${ }^{2}$

$$
\frac{d F(x)}{d x}=\max \{0,1-c\} \delta_{0}(x)+\frac{\sqrt{(x-a)(b-x)}}{2 \pi x} 1_{[a, b]}(x)
$$

where $a=(\sqrt{c}-1)^{2}, b=(\sqrt{c}+1)^{2}, \delta_{0}(x)$ is the Dirac's delta function centered at $x=0$ and

$$
1_{I}(x)= \begin{cases}1, & \text { if } x \in I \\ 0, & \text { otherwise }\end{cases}
$$

From the almost sure convergence of the distributions $F^{N}(x)$ toward $F(x)$ follows the convergence with probability one of $C$ in (2) to $N \cdot \bar{C}$, where

$$
\bar{C}=\bar{C}(\beta, c)=\int_{-\infty}^{\infty} \log _{2}\left(1+\frac{\beta}{c} x\right) d F(x)
$$

which is a nonrandom quantity that will be referred to as asymptotic capacity per antenna or spatial efficiency (number of bits per receive antenna that can be transmitted arbitrarily reliably). The integral in (4) can be computed in closed form, as shown in [6] and [7]. In this paper, we try to generalize that expression including the effect of correlation between the entries of $\mathbf{H}$ at either the transmitter or the receiver. This is motivated by the fact that, in practical mobile communication systems, the fading correlation between the array elements at the basestation can be made as low as desired by placing the antennas far from one another. This is more complicated in mobile stations, where the space limitations constitute a great impediment to achieving low fading correlations. This way, one can regard our model as the result from a typical communication scenario both in the uplink and the downlink, where the antennas at the basestation are far from one another while the elements of the array at the mobile station are confined to a more limited space.

\footnotetext{
${ }^{2}$ We make the abuse of representing $F(x)$ as a differentiable function (although it is clearly not whenever $c<1)$.
} 


\section{CAPACity Under Correlated Fading Model}

Recently, the evaluation of the MIMO capacity under a correlated fading model has received much attention. In the nonasymptotic scenario, several papers have considered the evaluation of the capacity under different channel models. In [11], the authors propose a scattering model and evaluate the capacity under different rank and correlation considerations of the channel matrix. A similar approach is also taken in [12]. On the other hand, in an attempt to avoid the need for simulations or the channel model assumption, several papers have considered and investigated bounds to the MIMO mean channel capacity under fading correlation. In [13], the authors obtain different interesting upper bounds to the mean capacity under uniform transmit power allocation and correlation at either the transmit or the receive side. These bounds were then evaluated under an abstract scatterer model and different antenna array configurations. Other bounds, based on the application of Jensen's inequality can be found in [14].

The main drawback of all these contributions is the fact that they are either based on numerical evaluations or they can only describe the behavior of the capacity in terms of bounds. Thus, in order to simplify the analysis and gain further insight into the problem, several studies have focused on the asymptotic capacity under the assumption of a high number of antennas at either the transmit or the receive side (or even both). In [15], the authors considered an asymptotic situation where the number of transmit antennas increases without bound while the number of receive antennas is held constant. The asymptotic distribution of the capacity under uniform power allocation was then evaluated assuming correlation at both the transmitter and the receiver. The same problem was also recently studied in [8] and [16], although here the asymptotic approximation considered a high number of antennas at both transmit and receive sides. Some other asymptotic investigations, also taking into account the fading correlation at both sides of the communication link, have been proposed in [17] and [18].

Our asymptotic study is similar to that in [8] and [16]. In [8], no particular form is imposed on the fading correlation functions and the capacity was to be the solution of an equation depending on these general functions. In [16], a channel model based on physical approximations is proposed and the asymptotic capacity for that model is evaluated using numerical integration. The main advantage of these two approaches resides in the fact that, since the quotient between transmit and receive antennas is held constant, the asymptotic capacity can be more representative in situations where the number of transmit antennas is of the same order of magnitude as the number of receive ones.

Next, we propose a similar approach to the problem, focusing on the case where fading correlation arises at the receiver only. Later, we will indicate how to translate these results to the situation with fading correlation at the transmitter.

\section{A. Correlation at the Receive Side}

Here, we will model the channel matrix $\mathbf{H}$ in (1) as

$$
\mathbf{H}=\mathbf{C}^{1 / 2} \mathbf{U}
$$

where $\mathbf{U}$ is an $N \times M$ matrix with i.i.d. circularly symmetric complex Gaussian entries with zero mean and unit variance and
C is an $N \times N$ Hermitian Toeplitz matrix that contains, at each of its entries, the fading correlation between two receive elements (here, $\mathbf{C}^{1 / 2}$ stands for the Hermitian positive definite square root of $\mathbf{C}$ ). Note that the Toeplitz assumption restricts the actual configuration of the system to a linear structure (for instance, a uniform linear array).

Consider the expression of the asymptotic capacity (4) but with $F(x)$ incorporating the fading correlation matrix at the receiver and let $\bar{C}^{\mathrm{RX}}$ denote the asymptotic capacity per receive antenna. Taking derivatives with respect to $\beta$, one obtains

$$
\begin{aligned}
\frac{\delta \bar{C}^{\mathrm{RX}}}{\delta \beta} & =\frac{1}{\log 2}\left[\frac{1}{\beta} \int_{-\infty}^{\infty} d F(x)-\frac{c}{\beta^{2}} \int_{-\infty}^{\infty} \frac{1}{\frac{c}{\beta}+x} d F(x)\right] \\
& =\frac{1}{\log 2}\left[\frac{1}{\beta}+\frac{c}{\beta^{2}} m\left(-\frac{c}{\beta}\right)\right]
\end{aligned}
$$

with $m(z)$ the Stieltjes transform of the distribution $F(x)$

$$
m(z)=\int_{-\infty}^{\infty} \frac{1}{z-x} d F(x) .
$$

As already suggested in [19], given an expression for the Stieltjes transform of the asymptotic distribution of eigenvalues of $N^{-1} \mathbf{H H}^{H}$, one can obtain the asymptotic capacity per antenna integrating (6) and forcing $\left.\bar{C}^{\mathrm{RX}}\right|_{\beta=0}=0$. The Stieltjes transform $m(z)$ will be derived using results from Free Probability Theory [4] and, in particular, the theory of multiplicative free convolution of measures. This is a useful tool that describes the eigenvalue distribution function of a product of infinite-dimensional matrices as a function of the eigenvalue distribution of each matrix (see [4] for more details). The key observation in our problem is that, as shown in [20], when the dimensions of the problem increase without bound, the two matrices $\{\mathbf{C}\},\left\{1 / N \mathbf{U} \mathbf{U}^{H}\right\}$ become asymptotically free almost surely (a concept similar to independence but applied to noncommutative random variables). Under these circumstances, one can obtain the asymptotic eigenvalue distribution function of the product of the two matrices from the asymptotic eigenvalue distribution of each one. This is achieved by means of the so-called S-transform (the noncommutative analog of the Mellin transform), which can be obtained from the Stieltjes transform defined above as

$$
S(z)=\frac{1+z}{z} \chi(z)
$$

with $\chi(z)$ the formal inverse of $\psi(z)$ (i.e., $\psi(\chi(z))=$ $\chi(\psi(z))=z)$

$$
\psi(z)=z^{-1} m\left(z^{-1}\right)-1
$$

and $m(z)$ as in (7). Both the Stieltjes transform and the S-transform have already been used to model the asymptotic multipath MIMO channel in [16], [21].

The importance of the S-transform stems from the fact that the S-transform of the density of eigenvalues of a product of two freely independent random matrices is equal to the product of S-transforms of each matrix, in our case

$$
S(z)=S_{U}(z) S_{C}(z)
$$

where $S_{U}(z)$ and $S_{C}(z)$ are the S-transforms of the densities of eigenvalues of $(1 / N) \mathbf{U} \mathbf{U}^{H}$ and $\mathbf{C}$, respectively. It can be 
shown [5] that the S-transform of the Marčenko-Pastur law is given by

$$
S_{U}(z)=\frac{1}{z+c}
$$

so that the S-transform of the final density takes the form

$$
S(z)=\frac{1}{z+c} S_{C}(z)
$$

At this point, one should choose a model for the fading correlation, obtain the S-transform of the corresponding asymptotic eigenvalue distribution $S_{C}(z)$, insert it into (8) and undo the transformation to get to the corresponding Stieltjes transform $m(z)$. From that point, one would obtain the asymptotic capacity per antenna expression by integration in (6). The only obstacle here is the fact that in order to get to the Stieltjes transform from the S-transform, one must find the formal inverse of $\chi(z)=(z / 1+z) S(z)$ and that can only be done with relative ease of manipulation when $\chi(z)$ is a quotient of secondorder polynomials in $z$. Of course, a closed expression can be given for that inverse even when $\chi(z)$ is a quotient of third- and fourth-order polynomials, but the analytical manipulation of the capacity expressions turns out to be very difficult. Thus, it seems advisable to model the asymptotic density of eigenvalues of $\mathbf{C}$ with a function that yields a first-order polynomial as S-transform. Such density can be described as a tilted semicircular law and takes the form ${ }^{3}$

$$
f_{C}(x)=\frac{1}{2 \pi \mu x^{2}} \sqrt{\left(\frac{x}{\sigma_{1}}-1\right)\left(1-\frac{x}{\sigma_{2}}\right)} 1_{\left[\sigma_{1}, \sigma_{2}\right]}(x)
$$

with support $\left[\sigma_{1}, \sigma_{2}\right]$ and where we have defined

$$
\mu=\frac{\left(\sqrt{\sigma_{2}}-\sqrt{\sigma_{1}}\right)^{2}}{4 \sigma_{1} \sigma_{2}}
$$

assuming of course $\mu>0$. Here, $\mu$ is a parameter that controls the degree of fading correlation: low values of $\mu$ correspond to uncorrelated fading models, so that, as $\mu$ grows large, the fading correlation between antennas becomes stronger.

We have two degrees of freedom to fix the support of $f_{C}(x)$, namely $\sigma_{1}, \sigma_{2}$. One will be used to normalize $\mathbf{C}$ (setting for instance its diagonal entries to one), while the other will be related to the interelement fading correlation, which is in turn associated with the eigenvalue spread of $\mathbf{C}$ (namely, a zero eigenvalue spread will be related to a zero-correlation model $\mathbf{C}=\mathbf{I}_{N}$ while higher spreads will be associated with higher correlation modes). To fix the asymptotic diagonal entries of the correlation matrix $\mathbf{C}$ to one, one must force the first-order moment of the density in (9) to one, i.e.,

$$
\int x f_{C}(x) d x=1
$$

This is because, since $\mathbf{C}$ is Toeplitz, fixing its diagonal entries to one is equivalent to fixing $(1 / N) \operatorname{tr}[\mathbf{C}]=1$. Hence, if $F_{C}^{N}(x)$

\footnotetext{
${ }^{3}$ It is important that the density chosen corresponds to a probability measure, as it is the case. Otherwise, one cannot use the theory of free convolution of measures.
}

denotes the eigenvalue empirical distribution function of the matrix with finite dimensions $(N \times N)$, one can express

$$
\frac{1}{N} \operatorname{tr}[\mathbf{C}]=\int x d F_{C}^{N}(x) .
$$

Now, since $F_{C}^{N}(x) \rightarrow F_{C}(x)$ with probability one and $F_{C}(x)$ is differentiable with derivative $f_{C}(x)$, one readily sees that $(1 / N) \operatorname{tr}[\mathbf{C}]$ tends almost surely, as $N \rightarrow \infty$, to the integral in (10). The integral itself can be computed in closed form and turns out to be equal to $\sigma_{1} \sigma_{2}$. Therefore, the normalization constraint in the asymptotic eigenvalue distribution of $\mathbf{C}$ will be expressed as $\sigma_{1} \sigma_{2}=1$. This leaves us with a single degree of freedom $(\mu)$ to parametrize the degree of correlation between antennas, which, after the normalization $\sigma_{1} \sigma_{2}=1$, can be expressed as

$$
\mu=\frac{\left(1-\sqrt{\frac{\sigma_{2}}{\sigma_{1}}}\right)^{2}}{4 \sqrt{\frac{\sigma_{2}}{\sigma_{1}}}} .
$$

Note that $\sqrt{\sigma_{2} / \sigma_{1}}$ is the asymptotic eigenvalue spread of the correlation matrix $\mathbf{C}$, which is higher than one by assumption. Let us now discuss how to choose $\mu$ in a practical situation.

Assume, for example, that we want to model an exponentially decaying correlation model, i.e., $\left\{\mathbf{C}^{\exp }\right\}_{i, j}=\rho^{|i-j|}$, with $0<$ $\rho<1$ the correlation parameter. It is well known that the asymptotic density of eigenvalues of a Toeplitz matrix can be extracted from the Fourier transform of the sequence of its skew-diagonal entries (assuming of course that the Fourier transform exists, cf. [22]). In our case, it can be shown that the density of eigenvalues of $\mathbf{C}^{\exp }$ has support $[1-\rho / 1+\rho, 1+\rho / 1-\rho]$. Thus, if we want to model an exponentially decaying fading model, we can fix

$$
\frac{\sigma_{2}}{\sigma_{1}}=\left(\frac{1+\rho}{1-\rho}\right)^{2}
$$

so that the distribution in (9) generates the same eigenvalue spread as the exponential model. This is equivalent to fixing $\mu$ in our distribution as

$$
\mu=\frac{\rho^{2}}{1-\rho^{2}} .
$$

Of course, fixing the support of the proposed distribution so as to guarantee the same eigenvalue spread of another model (such as the exponential) is not the only alternative. In fact, provided that we are trying to approximate a nonasymptotic situation, a more sensible approach would be to approximate the fading correlation function around the origin, fixing for instance $\sigma_{1} / \sigma_{2}$ (or, equivalently, $\mu$ ) to guarantee that the correlation between consecutive elements in the array coincides with a specified value, which will be denoted by $\lambda$. This value can be chosen from measurements - provided that we are trying to analyze a real channel—or fixed as the corresponding correlation generated by the distribution that we are trying to approximate with our model.

Assume once more that we want to approximate an exponentially decaying correlation model $\left(\mathbf{C}^{\exp }\right)$. Two different approximation alternatives have been presented so far. We have seen that $\mu$ can be fixed as in (12) to guarantee that the eigenvalue 

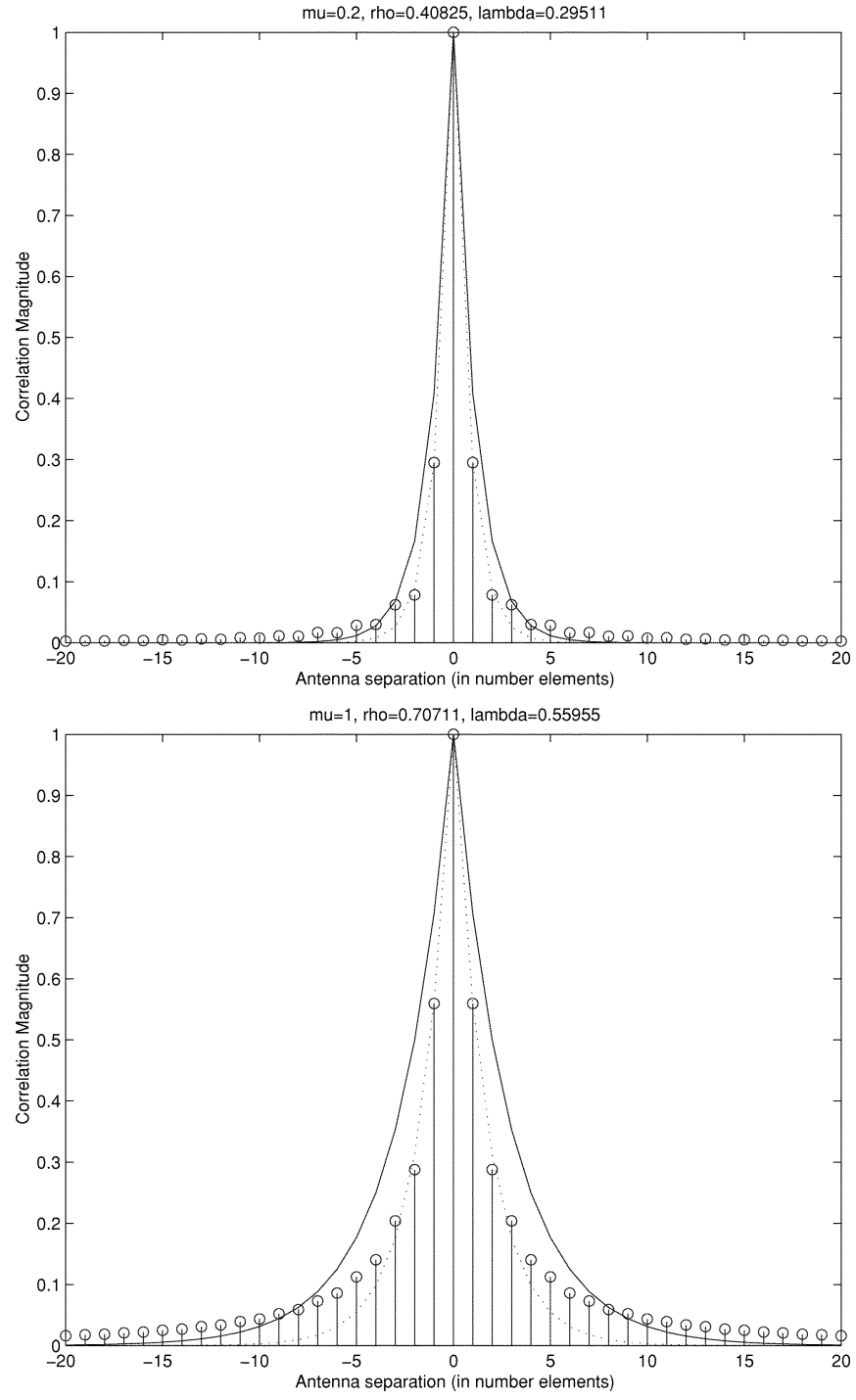

Fig. 1. Comparative representation of the shape of the proposed and the exponential correlation models. Stems: proposed model. Solid line: exponential model that generates the same eigenvalue spread of the covariance matrix (exponential correlation parameter denoted by rho). Dotted line: exponential model that generates the same correlation between consecutive elements (denoted by lambda).

spread of $\mathbf{C}^{\exp }$ is the same as the eigenvalue spread of $\mathbf{C}$. An alternative approach is to fix $\mu$ so that the correlation between consecutive elements of our model is the same as the correlation between consecutive elements in the exponential correlation case (which in turn is equal to $\rho$ ). In Fig. 1, we represent with stems, our proposed correlation model for $\mu=0.2$ and $\mu=1$ (see [22] for indications on how to obtain the actual correlation model from the density of eigenvalues). In solid line we represent the exponential correlation model that generates the same eigenvalue spread [fixing $\rho$ according to (12)] and in dotted line the exponential model that results in the same correlation between consecutive elements (fixing the exponential parameter to be equal to $\lambda$ ). It is observed that the exponential model generating the same eigenvalue spread is a good approximation of the global correlation function that we are proposing, whereas the exponential model that generates the same correlation between consecutive elements is more adjusted to the correlation shape around the origin. In any case, it is important to see

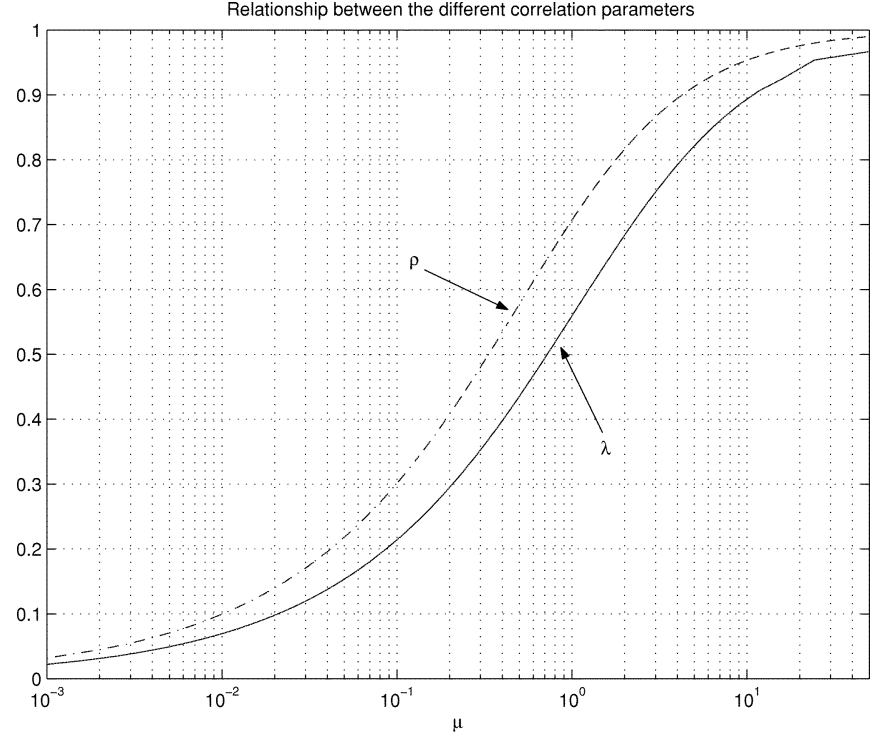

Fig. 2. Correlation between consecutive elements $\lambda$ as a function of the fading parameter $\mu$ and relationship with the exponential correlation parameter that yields the same eigenvalue spread $(\rho)$. For a given $\mu$, the parameter $\lambda$ gives the correlation between consecutive elements that the proposed model generates. The value of $\rho$ should be understood as the correlation parameter in an exponentially decaying model that results in the same eigenvalue spread as the proposed model.

that even though we are imposing a certain shape of the eigenvalue distribution, we can still accommodate a more realistic scenario through the parameter $\mu$.

To illustrate the range of values of $\mu$ that one can expect in a real situation, Fig. 2 represents the fading correlation between consecutive elements $(\lambda)$ and the exponential parameter $\rho$ in (12) as a function of the correlation parameter of the proposed model $(\mu)$. We observe that in practical situations the correlation parameter of the proposed model will oscillate between 0.01 and 1 (which generate an approximate correlation between consecutive elements of $\lambda \approx 0.05$ and $\lambda \approx 0.6$, respectively).

Now, returning to the derivation of the asymptotic capacity per antenna, the S-transform of the distribution $f_{C}(x)$ takes the form (see [5] for the derivation)

$$
S_{C}(z)=\frac{1}{\sqrt{\sigma_{1} \sigma_{2}}}-\frac{\left(\sqrt{\sigma_{2}}-\sqrt{\sigma_{1}}\right)^{2}}{4 \sigma_{1} \sigma_{2}} z=1-\mu z
$$

where we have introduced the normalization constraint $\sigma_{1} \sigma_{2}=1$. Inserting this expression in (8) and using the formulas above, one easily gets to the Stieltjes transform of the final global matrix

$$
m(z)=\frac{z+2 z \mu+1-c+\sqrt{[z-(1+c)]^{2}-4 c(1+\mu z)}}{2 z(1+\mu z)} .
$$

To obtain the asymptotic density of eigenvalues of the global matrix $N^{-1} \mathbf{H H}^{H}$, one can use the inverse Stieltjes transform presented in, e.g., [3], which gives

$$
\frac{d F(x)}{d x}=\max \{0,1-c\} \delta_{0}(x)+\frac{\sqrt{\left(x-\xi_{1}\right)\left(\xi_{2}-x\right)}}{2 \pi x(1+\mu x)} 1_{\left[\xi_{1}, \xi_{2}\right]}(x)
$$


where $\delta_{0}(x)$ and $1_{\left[\xi_{1}, \xi_{2}\right]}(x)$ are as defined in (3) and

$$
\begin{aligned}
& \xi_{1}=1+c+2 \mu c-2 \sqrt{c} \sqrt{(1+\mu)(1+\mu c)} \\
& \xi_{2}=1+c+2 \mu c+2 \sqrt{c} \sqrt{(1+\mu)(1+\mu c)} .
\end{aligned}
$$

From this point, one can obtain a closed expression for the asymptotic capacity per antenna integrating (6) with respect to $\beta$ and forcing $\left.\bar{C}^{\mathrm{RX}}\right|_{\beta=0}=0$. The capacity per antenna takes the form (see [5] for details)

$$
\begin{aligned}
\bar{C}^{\mathrm{RX}}(\beta, c, \mu)= & \log _{2}\left|\beta w^{\mathrm{RX}}(\beta, c, \mu)\right| \\
& +\frac{1}{\mu} \log _{2}\left|1-\mu c \cdot v^{\mathrm{RX}}(\beta, c, \mu)\right| \\
& -(c-1) \log _{2}\left|u^{\mathrm{RX}}(\beta, c, \mu)\right| \\
& \text { bps/Hz/receive antenna }
\end{aligned}
$$

where the expressions of $u^{\mathrm{RX}}, v^{\mathrm{RX}}$, and $w^{\mathrm{RX}}$ are given in (16), at the bottom of the page. Note that the expression obtained is quite similar to the one presented in [7], which can be recovered taking $\mu \rightarrow 0$.

\section{B. Correlation at the Transmit Side}

Let us now analyze the case where fading correlation is present at the transmit side. In this case, we model the channel matrix as

$$
\mathbf{H}=\mathbf{U C}^{1 / 2}
$$

where $\mathbf{C}$ now is an $M \times M$ Hermitian Toeplitz deterministic matrix with limiting eigenvalue distribution given by (9) with the normalization constraint imposed before $\left(\sigma_{1} \sigma_{2}=1\right)$. The capacity can be expressed as the expected value (in terms of the statistics of $\mathbf{U}$ ) of

$$
\begin{aligned}
C^{\mathrm{TX}} & =\log _{2} \operatorname{det}\left[\mathbf{I}_{N}+\frac{\beta}{M} \mathbf{U} \mathbf{C U}^{H}\right] \\
& =\log _{2} \operatorname{det}\left[\mathbf{I}_{M}+\frac{\beta}{M} \mathbf{C} \mathbf{U}^{H} \mathbf{U}\right] .
\end{aligned}
$$

Now, let us denote by $G^{M}(x)$ the empirical distribution function of the eigenvalues of the $M \times M$ matrix $\mathbf{C}(1 / M) \mathbf{U}^{H} \mathbf{U}$ (as opposed to $F^{N}(x)$ for the case of correlation at the receive side). The capacity with correlation at the transmit side can be expressed as the expectation of

$$
C^{\mathrm{TX}}=M \int \log _{2} \operatorname{det}[1+\beta x] d G^{M}(x) .
$$

As $M, N \rightarrow \infty$ the empirical distribution function $G^{M}(x)$ tends almost surely to $G(x)$, which corresponds to $F(x)$ replacing $c$ by $1 / c$ in (13). Therefore, as $M, N \rightarrow \infty$, the quantity $(1 / N) C^{\mathrm{TX}}$ will tend to

$$
\bar{C}^{\mathrm{TX}}(\beta, c, \mu)=c \bar{C}^{\mathrm{RX}}\left(\frac{\beta}{c}, \frac{1}{c}, \mu\right)
$$

with $\bar{C}^{\mathrm{RX}}(\beta, c, \mu)$ as in (15).

Taking now $\mu \rightarrow 0$, we recover the same expression for the two capacities per receive antenna, i.e., ${ }^{4}$

$$
\begin{aligned}
\lim _{\mu \rightarrow 0} \bar{C}^{\mathrm{RX}}(\beta, c, \mu)= & \lim _{\mu \rightarrow 0} \bar{C}^{\mathrm{TX}}(\beta, c, \mu) \\
= & c \log _{2}\left|\frac{-c+(c-1) \beta-\sqrt{R_{0}}}{2 c}\right|+ \\
& -\frac{1}{\log 2} \frac{c+(1+c) \beta-\sqrt{R_{0}}}{2 \beta} \\
& -\log _{2}\left|\frac{c+(c-1) \beta-\sqrt{R_{0}}}{2 \beta}\right|
\end{aligned}
$$

where $R_{0}=c^{2}+2 c(1+c) \beta+(1-c)^{2} \beta^{2}$. The same happens if the number of sensors is the same at both sides of the communication link $(c=1)$, i.e.,

$$
\begin{aligned}
\bar{C}^{\mathrm{RX}}(\beta, 1, \mu)= & \bar{C}^{\mathrm{TX}}(\beta, 1, \mu) \\
= & \log _{2}\left|\frac{(1+2 \mu)\left[1+2 \beta+\sqrt{R_{1}}\right]-2 \mu(\beta-\mu)}{2[1+\mu]^{2}}\right| \\
& +\frac{1}{\mu} \log _{2}\left|1-\mu \frac{1+2 \beta+2 \beta \mu-\sqrt{R_{1}}}{2 \beta[1+\mu]^{2}}\right|
\end{aligned}
$$

where now $R_{1}=1+4 \beta(1+\mu)$.

\section{Preliminary Analysis}

Having arrived at this point and before considering a rigorous analysis of the asymptotic capacity expressions, let us compare the expressions obtained in the last two sections. Our main motivation here is to investigate whether the effect of fading correlation is worse at the receiver or at the transmitter. Thus, in Fig. 3,

${ }^{4}$ This is the expression obtained in [6] and [7].

$$
\begin{aligned}
u^{\mathrm{RX}}(\beta, c, \mu) & = \begin{cases}\frac{c+(1-c) \beta+2 \mu c^{2}-\sqrt{R^{\mathrm{RX}}}}{2 c(\beta-\mu c)} & \beta \neq \mu c \\
-\frac{c(1+\beta)}{c+(1+c) \beta} & \beta=\mu c\end{cases} \\
v^{\mathrm{RX}}(\beta, c, \mu) & =\frac{c+\beta(1+c)-\sqrt{R^{\mathrm{RX}}}+2 \beta c \mu}{2 c \beta\left[1+(1+c) \mu+c \mu^{2}\right]} \\
w^{\mathrm{RX}}(\beta, c, \mu) & =\frac{(1+(1+c) \mu)\left[c+\beta(1+c)+\sqrt{R^{\mathrm{RX}}}\right]-2 c \mu(\beta-\mu c)}{2 c \beta\left[1+(1+c) \mu+c \mu^{2}\right]}
\end{aligned}
$$

and

$$
R^{\mathrm{RX}}=[c+(1+c) \beta]^{2}-4 c \beta(\beta-c \mu) .
$$




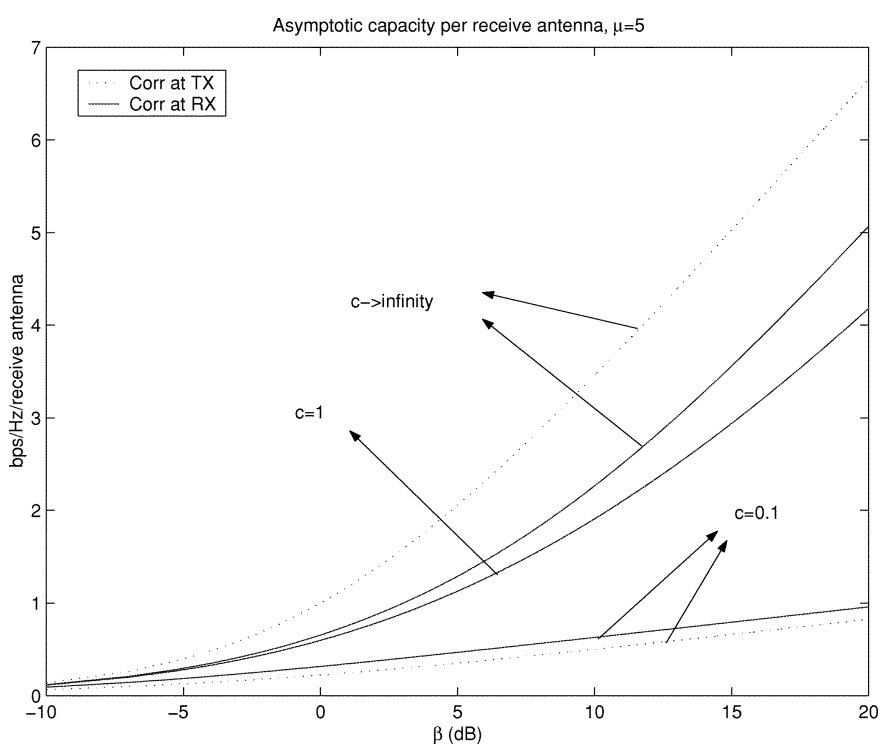

Fig. 3. Asymptotic capacity per receive antenna as a function of the signal to noise ratio for different values of the ratio $c=M / N$.

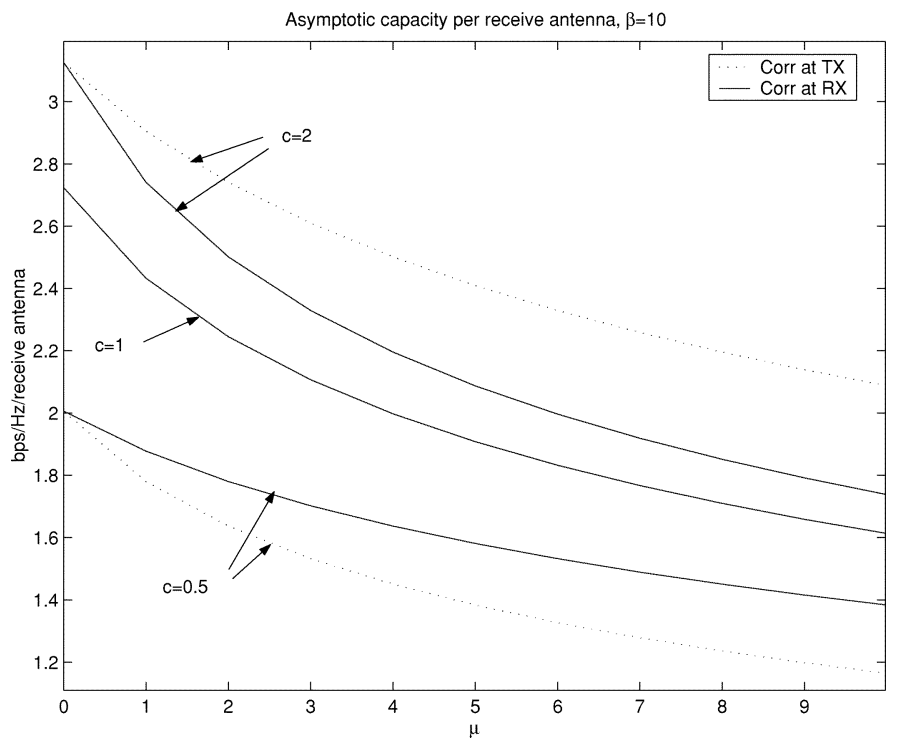

Fig. 4. Asymptotic capacity per receive antenna as a function of the correlation parameter $\mu$ for different values of the aspect ratio $c$.

we represent the asymptotic capacity per antenna as a function of the signal to noise ratio $\beta$ for different values of the aspect ratio $c$ fixing $\mu=5$ (this corresponds to a very high correlation between consecutive elements, i.e., $\lambda \approx 0.82$ ). The capacity for correlation at the transmitter is plotted in dotted line and the capacity obtained with correlation at the receiver is represented in solid line. Observe that when the number of transmit antennas is higher than the number of receive antennas $(c>1)$ the effect of fading correlation is worse at the receiver, while the opposite is true in the reverse situation. In conclusion, one can state the effect of fading correlation is more harmful at the side equipped with less antennas. In Fig. 4, we represent the asymptotic capacity per antenna as a function of the correlation parameter $\mu$ for different values of the aspect ratio $c$ fixing $\beta=10$. Note that fading correlation has always a detrimental effect and that its effect is more noticeable when it arises at the side with less antennas.

\section{AnAlysis OF THE RESUlts}

In this section, we analyze the influence of the correlation parameter $\mu$ on the spatial efficiency of the MIMO system. To homogenize the results with those presented in [6], we define the bit energy to noise spectral density at the receiver as

$$
\frac{E_{b}}{N_{0}}=\frac{\beta}{R}
$$

where $R$ is the data rate (information bits per receive antenna transmitted in a channel use). If the maximum efficiency is reached, one has

$$
\frac{E_{b}}{N_{0}}=\frac{\beta}{\bar{C}}
$$

where $\bar{C}$ is the asymptotic capacity per antenna $(\bar{C}$ represents either $\bar{C}^{\mathrm{TX}}$ or $\bar{C}^{\mathrm{RX}}$ depending on whether fading correlation is present at the transmit or receive side). One can express $\bar{C}$ as a function of $E_{b} / N_{0}$ denoted by $C^{\mathrm{TX}}$ and $C^{\mathrm{RX}}$ depending on whether transmit or receive correlation is modeled, by finding the solution to (15)-(18) replacing $\beta$ with $\left(E_{b} / N_{0}\right) C^{\mathrm{RX}}$ and $\left(E_{b} / N_{0}\right) C^{\mathrm{TX}}$, respectively, i.e.,

$$
\begin{aligned}
C^{\mathrm{RX}}= & \log _{2}\left|\frac{E_{b}}{N_{0}} C^{\mathrm{RX}} \cdot w^{\mathrm{RX}}\left(\frac{E_{b}}{N_{0}} C^{\mathrm{RX}}\right)\right| \\
& +\frac{1}{\mu} \log _{2}\left|1-\mu c \cdot v^{\mathrm{RX}}\left(\frac{E_{b}}{N_{0}} C^{\mathrm{RX}}\right)\right| \\
& -(c-1) \log _{2}\left|u^{\mathrm{RX}}\left(\frac{E_{b}}{N_{0}} C^{\mathrm{RX}}\right)\right|
\end{aligned}
$$

and equivalently for $C^{\mathrm{TX}}$.

Fig. 5 represents the asymptotic capacity per antenna as a function of $E_{b} / N_{0}$ for different values of the correlation parameter $\mu$ when $c=0.5$ and $c=2$. The simulated values of the correlation parameter (namely, $\mu=0,1,5$ ) correspond to a correlation between consecutive elements of $\lambda=0,0.56,0.82$. Note that the degradation of the asymptotic capacity per antenna does not seem very significant for typical values of the correlation parameter ( $\mu$ between 0.1 and 1 ).

Let us now analyze the general expression at high and low $E_{b} / N_{0}$ situations.

- Low $E_{b} / N_{0}$. The minimum $E_{b} / N_{0}$ required for reliable communication is not influenced by fading correlation, i.e.,

$$
\begin{aligned}
\left(\frac{E_{b}}{N_{0}}\right)_{\min } & =\lim _{\beta \rightarrow 0} \frac{\beta}{\bar{C}^{\mathrm{TX}}(\beta)}=\lim _{\beta \rightarrow 0} \frac{\beta}{\bar{C}^{\mathrm{RX}}(\beta)} \\
& =\log 2=-1.5917 \mathrm{~dB} .
\end{aligned}
$$

- High $E_{b} / N_{0}$. As $E_{b} / N_{0} \rightarrow \infty$, the slope of the achievable capacity per antenna with respect to $E_{b} / N_{0}$ tends to

$$
\begin{aligned}
\lim _{E_{b} / N_{0} \rightarrow \infty} \frac{C^{\mathrm{RX}}}{10 \log _{10}\left(\frac{E_{b}}{N_{0}}\right)} & =\lim _{\frac{E_{b}}{N_{0}} \rightarrow \infty} \frac{C^{\mathrm{TX}}}{10 \log _{10}\left(\frac{E_{b}}{N_{0}}\right)} \\
& =\left(10 \cdot \log 2 \cdot \log _{10} e\right)^{-1} \mathrm{~min}(c, 1) \\
& =0.3322 \mathrm{~min}(c, 1) \mathrm{bits} / \mathrm{dB}
\end{aligned}
$$



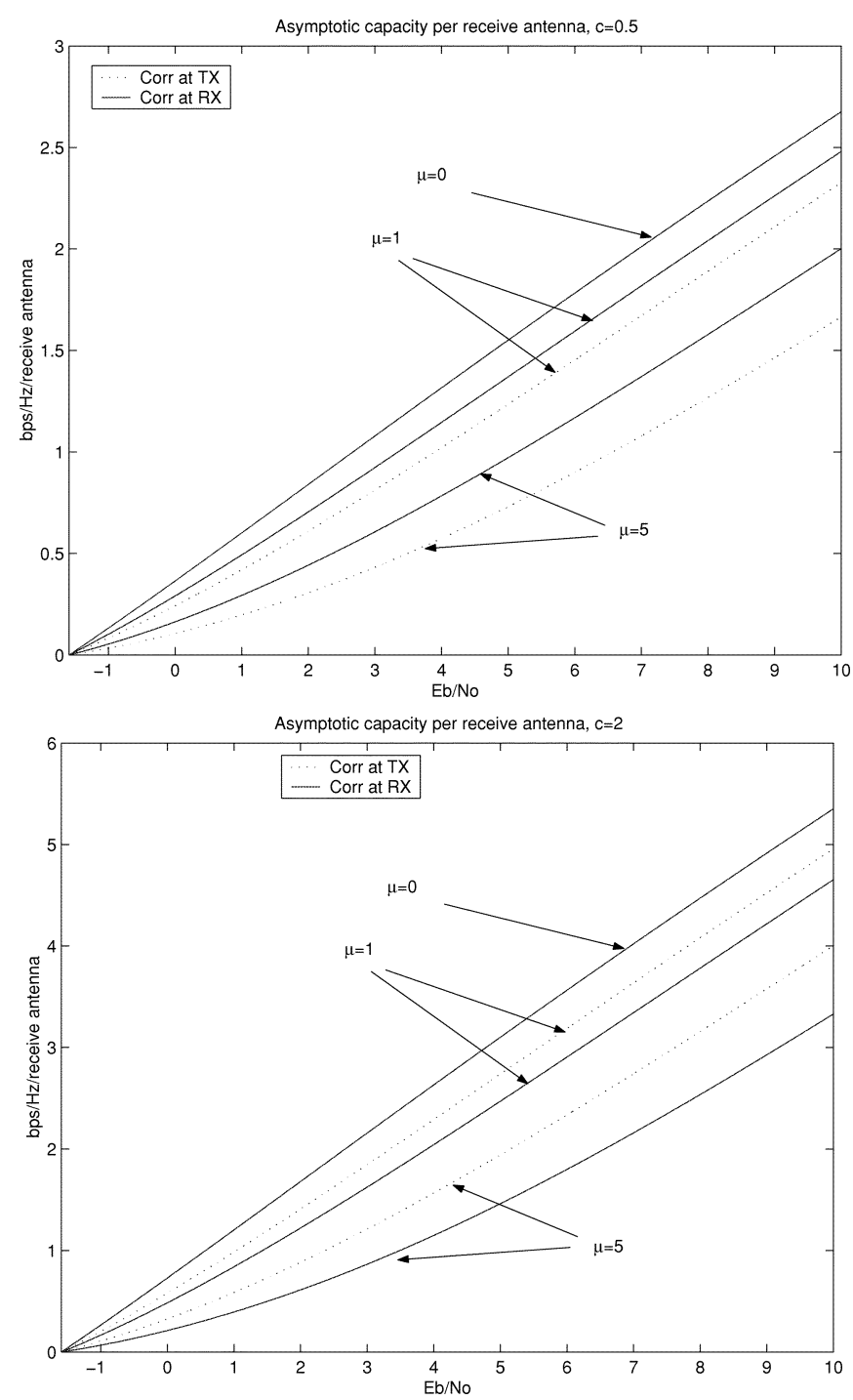

Fig. 5. Asymptotic capacity per receive antenna as a function of $E_{b} / N_{0}$ for different values of the correlation parameter $\mu$. In particular, the values $\mu=0,1,5$ correspond to a correlation between consecutive elements of $\lambda=0,0.56,0.82$, respectively.

which does not depend on the correlation coefficient $\mu$. Now let $C^{0}$ denote the capacity per receive antenna when $\mu=0$. The efficiency loss at high $E_{b} / N_{0}$ can be expressed as

$$
\begin{aligned}
& C^{0}-C^{\mathrm{RX}} \stackrel{\frac{E_{b}}{N_{0}} \rightarrow \infty}{\rightarrow} \begin{cases}\left(\frac{1}{\mu}+c\right) \log _{2}|1+\mu c|-\frac{c}{\log (2)} & c \leq 1 \\
\left(\frac{1}{\mu}+1\right) \log _{2}|1+\mu|-\frac{1}{\log (2)} & c>1\end{cases} \\
& C^{0}-C^{\mathrm{TX}} \stackrel{\frac{E_{b}}{N_{0}} \rightarrow \infty}{\rightarrow} \begin{cases}c\left(1+\frac{1}{\mu}\right) \log _{2}|1+\mu|-\frac{c}{\log 2} & c \leq 1 \\
\left(\frac{c}{\mu}+1\right) \log _{2}\left|\frac{c+\mu}{c}\right|-\frac{1}{\log 2} & c>1 .\end{cases}
\end{aligned}
$$

In Fig. 6, we represent the loss in capacity per antenna as a function of the correlation parameter $\mu$ for different values of the ratio $c$. Note that, if fading correlation is present at the receiver, the loss is maximum when the number of transmit antennas is higher than (or equal to) the number of receive antennas, $c \geq 1$. On the other hand, if fading correlation is present at the transmitter, the loss always in-
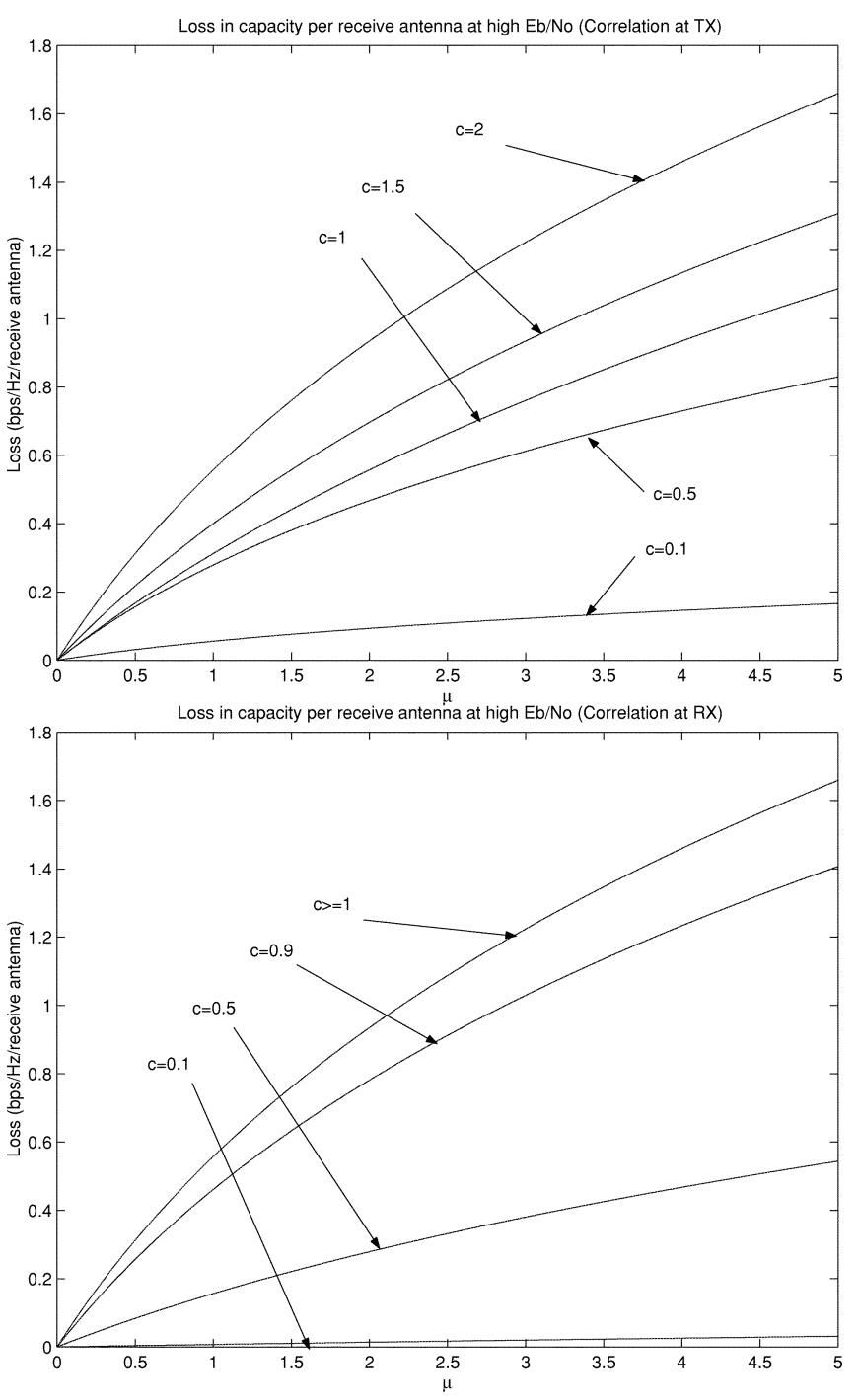

Fig. 6. Loss in capacity per receive antenna in presence of fading correlation.

creases with $c$. In any case, we see that the relative penalty introduced vanishes with $E_{b} / N_{0}$, i.e.,

$\lim _{E_{b} / N_{0} \rightarrow \infty} \frac{C^{0}-C^{\mathrm{RX}}}{C^{0}}=\lim _{E_{b} / N_{0} \rightarrow \infty} \frac{C^{0}-C^{\mathrm{TX}}}{C^{0}}=0$.

In conclusion, at high $E_{b} / N_{0}$ the influence of fading correlation is stronger when the number of transmit antennas is higher than the number of receive ones (irrespective of whether the fading correlation arises at the transmitter or the receiver).

Fig. 7 represents the capacity per antenna as a function of the ratio $c$ for different values of the correlation parameter $\mu$. Observe that as $c$ grows, the capacity per antenna saturates to a constant value that depends on the correlation parameter. If the fading correlation is present at the transmitter, this limit (denoted here by $C_{\infty}^{\mathrm{TX}}=\lim _{c \rightarrow \infty} C^{\mathrm{TX}}$ ) is the same regardless of the correlation parameter $\mu$ and is given as the nontrivial solution to the equation

$$
C_{\infty}^{\mathrm{TX}}=\log _{2}\left|1+\frac{E_{b}}{N_{0}} C_{\infty}^{\mathrm{TX}}\right| .
$$




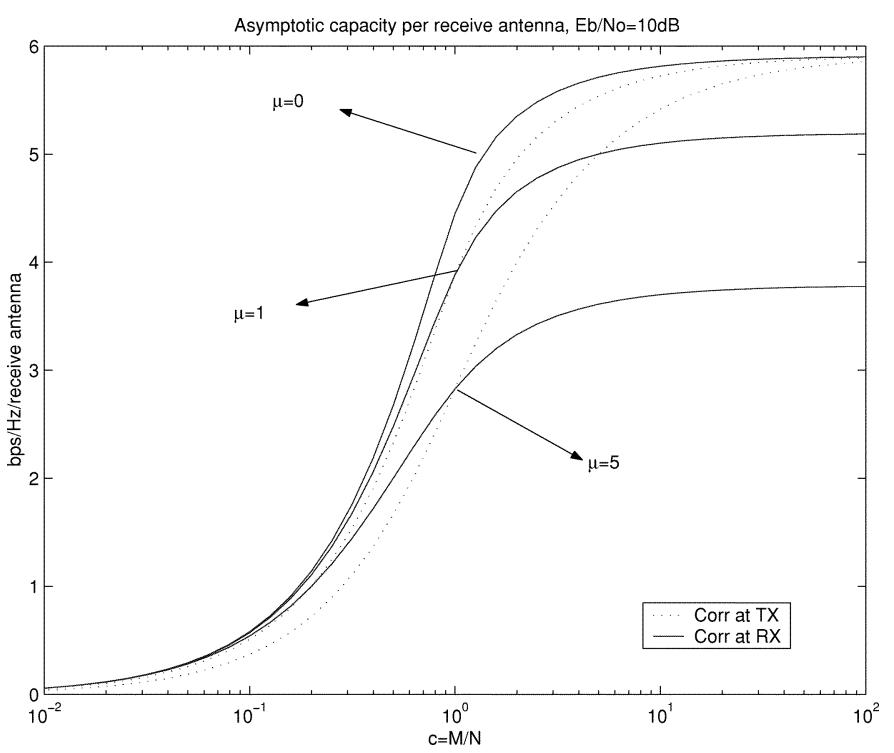

Fig. 7. Asymptotic capacity per antenna for $E_{b} / N_{0}=10 \mathrm{~dB}$.

This coincides with the asymptotic value presented in [6]. Conversely, if the fading correlation is present at the receiver, the capacity per antenna levels off to a constant value that depends on the correlation parameter $\mu$. Denote $C_{\infty}^{\mathrm{RX}}=\lim _{c \rightarrow \infty} C^{\mathrm{RX}}$. One easily finds that $C_{\infty}^{\mathrm{RX}}$ is the nontrivial solution to the equation

$$
\begin{aligned}
C_{\infty}^{\mathrm{RX}}= & \log _{2}\left|\frac{1+2 \mu+\beta_{\infty}+\sqrt{\left[1+\beta_{\infty}\right]^{2}+4 \beta_{\infty} \mu}}{2[1+\mu]}\right| \\
& +\frac{1}{\mu} \log _{2}\left|\frac{\beta_{\infty}-1+\sqrt{\left[1+\beta_{\infty}\right]^{2}+4 \beta_{\infty} \mu}}{2 \beta_{\infty}[1+\mu]}\right| \\
& -\frac{1}{\log 2} \frac{\beta_{\infty}+1-\sqrt{\left[1+\beta_{\infty}\right]^{2}+4 \beta_{\infty} \mu}}{2 \mu}
\end{aligned}
$$

where $\beta_{\infty}=\left(E_{b} / N_{0}\right) C_{\infty}^{\mathrm{RX}}$.

\section{NUMERICAL VALIDATION}

In this section, we validate the asymptotic capacity formulas obtained above via simulation. In Figs. 8 and 9, we represent in solid line the mean value of the capacity per antenna with the proposed correlation model as a function of the number of receive antennas for a fixed ratio $c$. The efficiency obtained with an exponential model with the same fading correlation between consecutive elements is plotted with asterisks and the predicted asymptotic value is represented in dotted line. The mean value of the simulated capacity per antenna was obtained averaging $10^{4}$ independent realizations of (1), modeling the entries of the channel matrix as circularly symmetric Gaussian random variables with zero mean, variance $10-\mathrm{dB}$ higher than the noise power and mutual relative covariance given by the corresponding model. We only represent the results for correlation at the receiver (the other case yields almost the same plots with a scale factor variation).

Note that the proposed and the exponential correlation models yield almost identical values of the capacity per

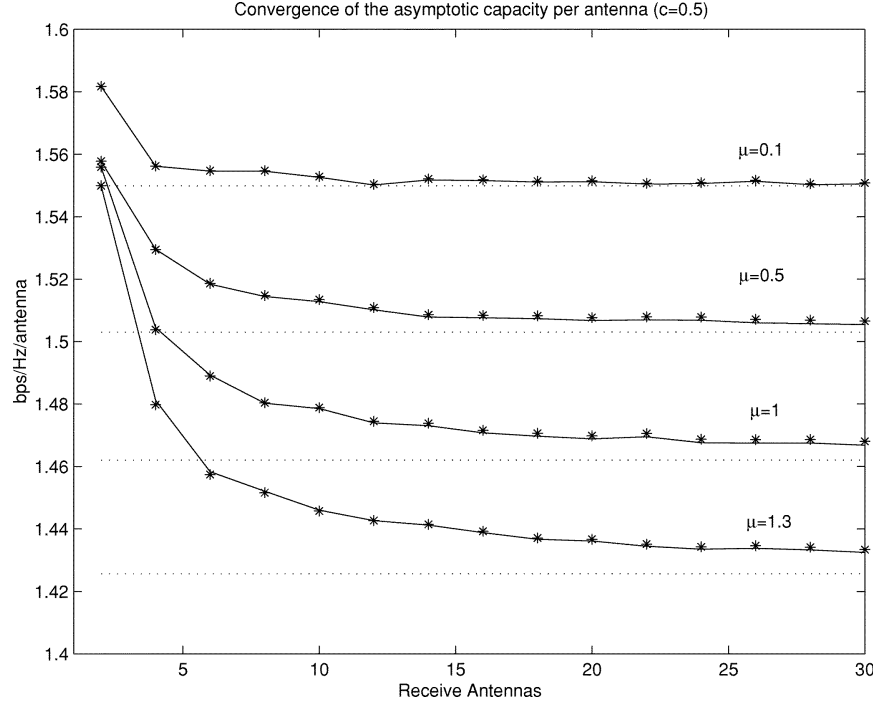

Fig. 8. Convergence of the mean value of the capacity per antenna toward its asymptotic value for $c=0.5$. Dotted lines: asymptotic efficiency. Solid lines: simulation with the proposed correlation model. Asterisks: simulation with exponential model with the same correlation between consecutive elements.

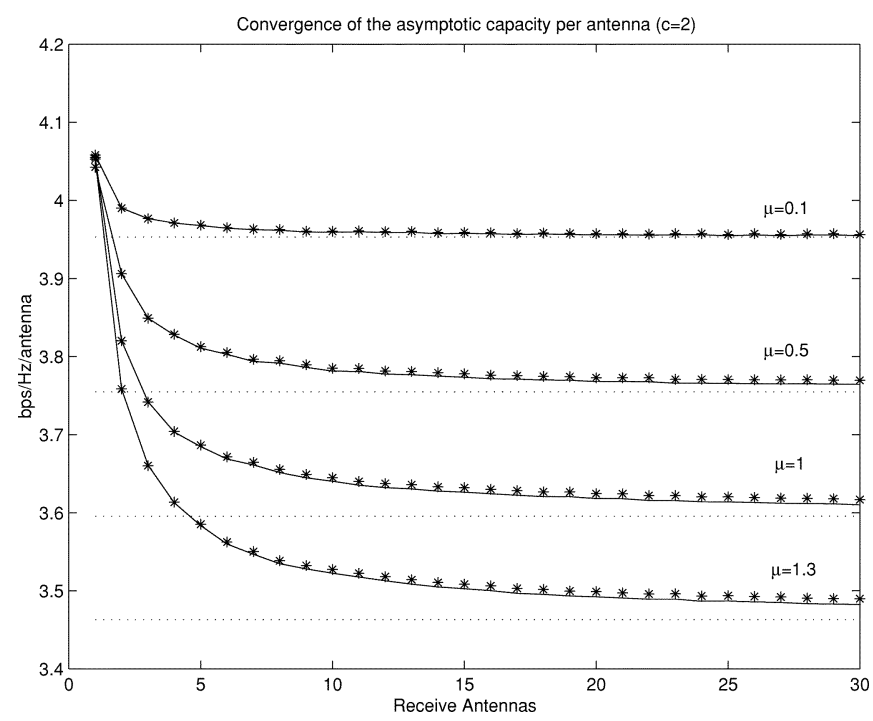

Fig. 9. Convergence of the mean value of the capacity per antenna toward its asymptotic value for $c=2$. Dotted lines: asymptotic efficiency. Solid lines: simulation with the proposed correlation model. Asterisks: simulation with exponential model with the same correlation between consecutive elements.

antenna and that the asymptotic expressions are always more pessimistic than the reality. On the other hand, the convergence is very fast for low values of the correlation parameter $\mu$ and gets slower as the interelement fading correlation increases.

\section{CONCLUSION}

We have presented a closed form expression of the asymptotic uniform power allocation capacity of a MIMO system assuming correlated fading at one side of the communications link. The expression is obtained modeling the asymptotic eigenvalue distribution of the fading correlation matrix as a tilted semicircular law depending on a parameter that describes the degree of fading correlation between antennas (at either the transmit or receive side). It has been shown that the proposed model is close 
to an exponentially decaying function and it has been demonstrated via simulation that, fixing the same correlation between consecutive elements, the two models yield almost the identical nonasymptotic capacity per antenna. The analytical expression obtained has been used to investigate several interesting properties of the asymptotic spatial efficiency and its relationship with the fading correlation. For instance, we have seen that, according to our model, fading correlation does not influence the rate of growth with $E_{b} / N_{0}$ (at high values of $E_{b} / N_{0}$ ) and its effect is more harmful at the side with less number of antennas. We have also seen that as the number of transmit antennas increases without bound and the number of receive antennas is held constant, the asymptotic spatial efficiency saturates to a constant value that depends on the fading correlation parameter only.

\section{ACKNOWLEDGMENT}

The authors would like to thank the anonymous reviewers for their helpful suggestions.

\section{REFERENCES}

[1] G. J. Foschini and M. J. Gans, "On limits of wireless communications in a fading environment when using multiple antennas," Wireless Pers. Commun., vol. 6, pp. 311-335, 1998.

[2] I. E. Telatar, "Capacity of multi-antenna Gaussian channels," Eur. Trans. Telecommun., vol. 10, no. 6, pp. 585-595, 1999.

[3] D. V. Voiculescu, K. J. Dykema, and A. Nica, "Free Random Variables," American Mathematical Society, vol. 1, CRM Monograph Series (Université de Montréal), 1992.

[4] D. V. Voiculescu, "Lectures on free probability theory," in Lecture Notes in Mathematics, P. Bernard, Ed. Berlin, Germany: Springer-Verlag, 2000, pp. 280-349.

[5] X. Mestre, "Space Processing and Channel Estimation: Performance Analysis and Asymptotic Results," Ph.D. dissertation, Universitat Politècnica de Catalunya, Spain, 2002.

[6] S. Verdú and S. Shamai, "Spectral efficiency of CDMA with random spreading," IEEE Trans. Inform. Theory, vol. 45, no. 2, pp. 622-640, Mar. 1999.

[7] P. B. Rapajic and D. Popescu, "Information capacity of a random signature multiple-input multiple-output channel," IEEE Trans. Commun., vol. 48, pp. 1245-1248, Aug. 2000.

[8] C.-N. Chuah, D. N. C. Tse, J. M. Kahn, and R. A. Valenzuela, "Capacity scaling in MIMO wireless systems under correlated fading," IEEE Trans. Inform. Theory, vol. 48, no. 3, pp. 637-650, March 2002.

[9] A. Lozano and A. M. Tulino, "Capacity of multiple-transmit multiplereceive antenna architectures," IEEE Trans. Inform. Theory, vol. 48, pp. 3117-3128, Dec. 2002

[10] U. Haagerup and S. Thorbjørnsen. (1998) Random Matrices With Complex Gaussian Entries. Centre for Mathematical Physics and Stochastics (Dept. Math. Sci.), Univ. Aarhus, Odebse M, Denmark. [Online]Tech. Rep. MSP-RR 1998-4. [Online]. Available: http://www. imada.sdu.dk/ haagerup/

[11] D. Gesbert, H. Bolcskei, D. Gore, and A. Paulraj, "MIMO wireless channels: Capacity and performance prediction," in Proc. Global Telecommunications Conf. GLOBECOM, vol. 2, 2000, pp. 1083-1088.

[12] A. M. Sayeed, "Modeling and capacity of realistic spatial MIMO channels," in Proc. IEEE Int. Conf. Acoustics, Speech, Signal Processing, Salt Lake City, UT, 2001, pp. 2489-2492.

[13] D. Shiu, G. J. Foschini, M. J. Gans, and J. M. Kahn, "Fading correlation and its effect on the capacity of multielement antenna systems," IEEE Trans. Commun., vol. 48, pp. 502-513, Mar. 2000.

[14] S. Loyka and A. Kouki, "New compound upper bound on MIMO channel capacity," IEEE Commun. Lett., vol. 6, no. 3, pp. 96-98, Mar. 2002 .
[15] C. Martin and B. Ottersten, "Asymptotic eigenvalue and capacity distributions for MIMO channels under correlated fading," IEEE J. Select. Areas Commun., submitted for publication.

[16] R. R. Müller, "A random matrix model of communication via antenna arrays," IEEE Trans. Inform. Theory, vol. 48, pp. 2495-2506, Sept. 2002.

[17] A. L. Moustakas, S. H. Simon, and A. M. Sengupta. (2002) MIMO Capacity Through Correlated Channels in the Presence of Correlated Interfers and Noise: A (not so) Large N Analysis. [Online]. Available: http://mars.bell-labs.com/

[18] A. M. Sengupta and P. P. Mitra. Capacity of Multivariate Channels With Multiplicative Noise: I. Random Matrix Techniques and Large-N Expansions for Full Transfer Matrices. [Online]. Available: http://arxiv.org/abs/physics/0010081

[19] S. Shamai and S. Verdú, "The impact of frequency-flat fading on the spectral efficiency of CDMA," IEEE Trans. Inform. Theory, vol. 47, no. 4, pp. 1302-1327, May 2001.

[20] F. Hiai and D. Petz, "The Semicircle Law. Free Random Variables and Entropy," American Mathematical Society, Providence, RI, 2000.

[21] R. R. Müller, "On the asymptotic eigenvalue distribution of concatenated vector-valued fading channels," IEEE Trans. Inform. Theory, vol. 48, pp. 2086-2091, July 2002.

[22] R. M. Gray, "Toeplitz and Circulant Matrices: A Review," Information Systems Laboratory, Stanford Univ., Stanford, CA, 1971.

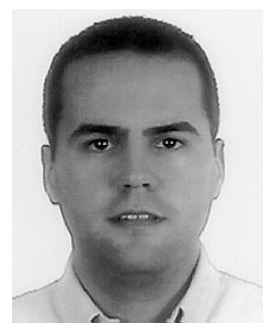

Xavier Mestre (S'96) was born in Barcelona, Catalonia, Spain, in 1974. He received the B.S. degree in electrical engineering from the Universitat Politècnica de Catalunya (UPC), Barcelona, Spain, in 1998.

In 1998, he joined the Department of Signal Theory and Communications, Universitat Politècnica de Catalunya, where he worked as a Research Assistant until December 2002. From January 2003, he is with the Centre Tecnològic de Telecomunicacions de Catalunya (CTTC), Spain.

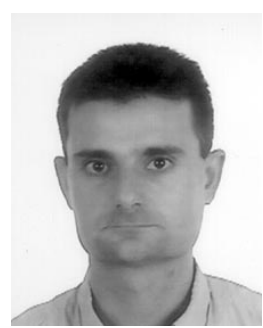

Javier R. Fonollosa (S'90-M'92-SM'98) received the $\mathrm{Ph} . \mathrm{D}$. degree in electrical and computer engineering from Northeastern University, Boston, MA, in 1992. From the Fall of 1989 until 1992, he was a Research Assistant at Northeastern University, Boston, MA and the University of Southern California, Los Angeles, CA, where upon completion of the Ph.D. degree, he became Research Associate.

In 1989, he held a visiting appointment at the European Space Agency Centre, The Netherlands. In 1993, he joined the Department of Signal Theory and Communications, Universitat Politècnica de Catalunya, Barcelona, Spain, where he is an Associate Professor since November 1996.

Dr. Fonollosa has been a Member of the Signal Processing for Communications (SPCOM) Technical Committee since 1998.

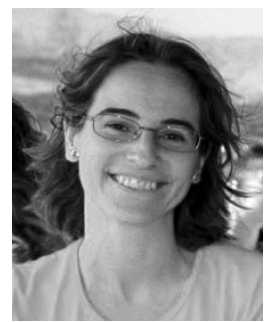

Alba Pagès-Zamora received the M.S. and Ph.D. degrees in electrical engineering from the Universitat Politècnica de Catalunya (UPC), Barcelona, Spain, in 1992 and 1996, respectively.

In 1992, she joined the Department of Signal Theory and Communications at the same University and became an Associate Professor in 2001. She teaches graduate and undergraduate courses related to statistical signal processing. Her current research interests include array signal processing, location in wireless systems, and MIMO channel wireless 